\title{
Bilanz ziehen:
}

\section{Zahlen, Daten} und Fakten in der Pädiatrie

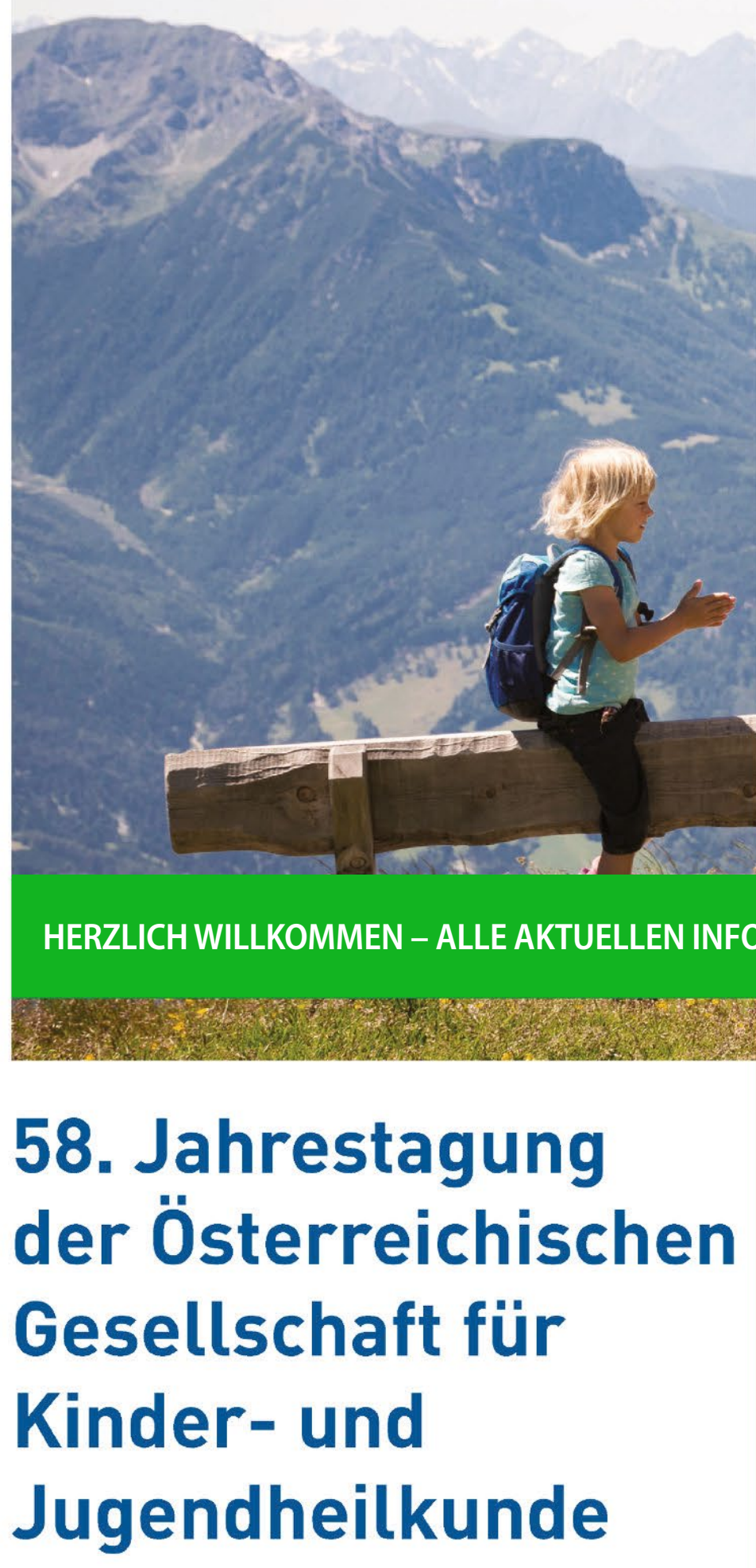

(2) ÖSTERREICHISCHE GESELLSCHAFT
FÜR KINDER- UND JUGENDHEILKUNDE

INFORMATIONEN UNTER www.oegkj-jahrestagung.at as atser
24.-26.
September
2020
Congress
Innsbruck 


\section{Mitteilungen der ÖGKJ}

Monatsschr Kinderheilkd 2020 • 168:661-668

https://doi.org/10.1007/s00112-020-00967-z

(c) Springer Medizin Verlag GmbH, ein Teil von Springer Nature 2020

\section{Redaktion (V.i.S.d.P.)}

Prof. Dr. Reinhold Kerbl

LKH Hochsteiermark/Leoben

Abteilung für Kinder und Jugendliche

Vordernbergerstraße 42

A-8700 Leoben

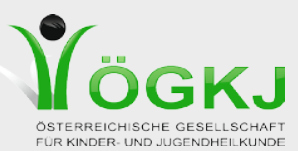

Präsidentin

Prof. Dr. Daniela Karall, Innsbruck

\section{Sekretärin}

PD Dr. Sabine Scholl-Bürgi, Innsbruck

Geschäftsstelle der ÖGKJ für Öffentlichkeitsarbeit und Standesführung

Ralph Kerschbaumer

c/o S12! studio12 gmbh

Kaiser Josef Straße 9, A-6020 Innsbruck

Tel.: +43 512 890438 · Fax: + 43 512-890438-15

E-Mail: office@studio12.co.at·Internet: www.paediatrie.at

\section{Inhalt}

662 Aktuelles zur 58. ÖGKJ Jahrestagung in Innsbruck

663 Alles über Covid-19

663 Influenza-Impfung für Kleinkinder im Gratisimpfprogramm

664 Österreichs Angebot an Zentren für Kinder- und Jugendrehabilitation

666 Geburtstage

666 4. Österreichische Jahrestagung für Pädiatrische Psychosomatik

\section{Aktuelles zur 58. ÖGKJ Jahrestagung in Innsbruck}

\subsection{9. bis 26.09 .2020}

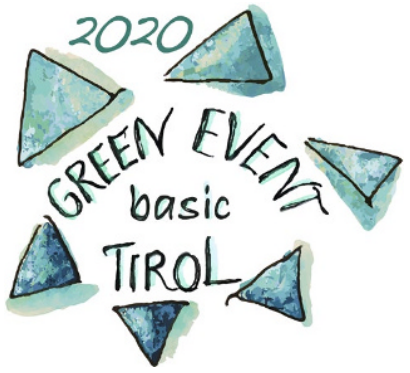

Liebe Kolleginnen und Kollegen!

Wenn Sie diese Ausgabe in Händen halten, dann können wir mit Sicherheit sagen, dass besondere Zeiten hinter uns - möglicherweise auch noch vor uns - liegen.

Soweit wir es erwarten (Stand 20.06.2020), wird die Jahrestagung heuer in Innsbruck auf jeden Fall vom 24.09. bis 26.09.2020 stattfinden, voraussichtlich mit einigen Auflagen, auf die wir bisher nicht so bewusst geachtet haben (z. B. bestimmte maximale Anzahl der Teilnehmer pro Raum, Abstand halten, u. ä.), und die wir Ihnen/Euch jeweils mitteilen werden.

\section{Also: Wird die Jahrestagung heuer stattfinden? Ja!}

Das Hauptthema der ÖGKJ Jahrestagung ist „Bilanz ziehen“ - Zahlen, Daten, Fakten in der Pädiatrie.

Insgesamt wird es in vier Plenarsitzungen Vorträge zu den Themen: Fazit aus epidemiologischen Erhebungen, Pädiatrie in einer geänderten Zeit (Teil 1 und 2) und Update zu neuen Therapien geben.

Daneben werden sieben Parallelsitzungen gestaltet.
Vier weitere Parallelsitzungen sind für insgesamt 24 Kurzvorträge reserviert. NEU ist dabei, dass es zur Einführung in die Kurzvorträge einen Impulsvortrag geben soll. Eine dieser Sitzungen ist zudem, wenn es die eingereichten Abstracts zulassen, ausdrücklich der Präsentation von Fallberichten gewidmet.

Zusätzlich wird wieder ein Niedergelassenen-Track mit für die niedergelassenen PädiaterInnen relevanten Themen und ein Facharzt-Prüfungsvorbereitungskurs abgehalten.

Am Samstag, 26.09., werden verschiedene Workshops angeboten.

NEU ist, dass Poster-Präsentationen in diesem Jahr in einer gemeinsamen Sitzung direkt nach der Eröffnung unter dem Motto „Poster und Drinks" stattfinden.

NEU ist auch, dass dem Thema Nachhaltigkeit/Umweltschutz bei ÖGKJ Jahrestagungen mehr Gewicht gegeben wird. Die Tagung ist als Green Event zertifiziert. D.h., dass bereits heuer einige Punkte konkret gelten:

1. Auf den Druck des Jahrestagungsprogrammes und des Abstractbandes wird verzichtet. Beide werden als PDF-File vor der Jahrestagung auf der Homepage (https://www.oegkj-jahrestagung.at/) und auch in der Jahrestagungs-APP verfügbar sein. Wer ein papierenes ausgedrucktes Programm haben möchte, muss es bitte zur Tagung selber mitbringen.

2. Auf eine Kongresstasche mit Werbeeinlagen wird verzichtet.

3. Um Anreise mit öffentlichen Verkehrsmitteln wird gebeten.

4. Regionales und saisonales Catering ist vorgesehen (Die Tiroler Küche hat da einiges zu bieten!)

Auch wenn es eine Umstellung bedeuten wird, die ja im Hinblick auf die ablaufende Corona-Krise ohnehin schon erforderlich ist:

Im Namen der TagungsorganisatorInnen - herzliche Einladung! Wir freuen uns sehr, Sie/Euch im September in Innsbruck begrüßen zu dürfen!

Herzliche Grüße,

\section{Daniela Karall}

Sabine Scholl-Bürgi

Innsbruck, 20.06.2020

Die jeweils aktualisierten Informationen zum Haupt- und RahmenProgramm sind auf der Homepage www.oegkj-jahrestagung.at oder auch www.paediatrie.at zu finden. 


\section{Alles über Covid-19}

\section{website Updates auf www.paediatrie.at}

Die ÖGKJ hat als Service für ihre nunmehr mehr als 1800 Mitglieder unter Federführung der AG Infektiologie (Volker Strenger) und unseres Generalsekretärs Reinhold Kerbl sehr rasch einen Informationsservice zum Thema Covid-19 implementiert.

Unter dem Button „Alles über COVID-19“ (www.paediatrie. at/covid) sind auf der Website der Gesellschaft mehrere Bereiche zum derzeit alles bestimmenden Thema zusammengefasst: neben dem wöchentlich aktualisierten Editorial und aktuellen Zahlen des Dashboards des Sozial- bzw. Gesundheitsministeriums finden sich Handlungsempfehlungen für pädiatrische Ordinationen, Links zu diverser aktueller Literatur und Studien sowie Stellungnahmen der ÖGKJ.

Diese Stellungnahmen der ÖGKJ wurden zum Einen den österreichischen Ministerien zur Verfügung gestellt - und fanden auch weitgehend in der politischen Entscheidungsfindung ihren Widerhall - zum Zweiten war es Ziel der Gesellschaft, damit auch die Öffentlichkeit über Presseaussendungen an nationale und regionale Medien zu informieren.

Die zahlreichen Zugriffe zeigen, dass dieser Service seitens der Mitglieder geschätzt und auch stark genutzt wird, daher werden die Inhalte weiterhin evaluiert und regelmäßig aktualisiert.

Verfasser:

Ralph Kerschbaumer, Innsbruck

\section{Influenza-Impfung für Kleinkinder im Gratisimpfprogramm}

\section{Bericht von einer Webkonferenz am 19.05.2020}

Das Zusammentreffen weiterer Corona-Infektionen mit der Influenzasaison 2020/21 könnte für die Kinder- und Jugendgesundheit ein ernsthaftes Problem erbringen. Die Österreichische Gesellschaft für Kinder- und Jugendheilkunde (ÖGKJ) hat daher eingefordert, die Influenzaimpfung zumindest für die nächste Saison kostenfrei zur Verfügung zu stellen. Seitens des Gesundheitsministeriums wurde diesbezüglich Gesprächsbereitschaft signalisiert.

Am 19.05.2020 fand ein Meinungsaustausch zwischen dem Gesundheitsministerium und Vertretern der ÖGKJ statt. Wie in Corona-Zeiten üblich, erfolgte dieser im Rahmen einer Webkonferenz. Bedauerlicherweise musste sich BM Anschober kurzfristig entschuldigen, wurde aber durch den Kommissär Mag. V. Rest vertreten. Schließlich nahmen folgende Personen an der Videokonferenz teil:

Seitens des Ministeriums:

Mag. Vinzent REST (Kommissär im Ministerium für Soziales, Gesundheit, Pflege und Konsumentenschutz)

SC Dr. Silvia TÜRK (Leiterin der Sektion VIII, Gesundheitssystem)
Mag.Dr. PAULKE-KORINEK, PhD (Abteilung VIII/10/C, Leitung Impfwesen)

Seitens der ÖGKJ:

A.Univ.Prof. Dr. Daniela KARALL (Präsidentin)

Univ.Prof. Dr. Reinhold KERBL (Generalssekretär)

Privatdozent Dr. Hans Jürgen DORNBUSCH (Impfreferent)

Univ.Prof. Dr. Werner ZENZ (Pädiatrische Influenza Task Force)

In der Webkonferenz wurden zunächst die möglichen Probleme des Zusammentreffens von Influenza- und Corona-Infektionen dargestellt. Diese sind:

- Problem der Differenzierung in der Diagnostik

- Mögliche Überlastung des Gesundheitssystems, insbesondere der niedergelassenen Kinder-und JugendfachärztInnen sowie der pädiatrischen Abteilungen

- Das Zusammentreffen von Influenza-und Corona-Doppelinfektionen könnte zu sehr schweren Verläufen führen

- Im Unterschied zu Corona-Infektionen sind schwere Verläufe der Influenza relativ häufig (z. B. 9 Todesfälle in der Influenzasaison 2017/18 )

- wahrscheinlich anders als bei „Corona“ sind Kinder für Influenza auch relevante „Spreader“

Seitens des Ministeriums wurde große Bereitschaft signalisiert, die Influenzaimpfung in der Saison 2020/21 kostenfrei zur Verfügung zu stellen. Es wurden für diese Saison 200.000 Impfdosen des intranasalen Impfstoffes Fluenz Tetra ${ }^{\oplus}$ bestellt. Noch nicht klar ist, ob dieser Impfstoff seitens der Firma (Astra Zeneca) auch in diesem Ausmaß nach Österreich geliefert werden kann. Als Alternative wurde daher die intramuskuläre Vakzine vorgeschlagen.

Gemeinsam wurden folgende Absichtserklärungen abgegeben:

- Kostenfreie Influenza-Impfung für Klein- und Vorschulkinder im Alter von 2-6 Jahren

- Bevorzugter Impfstoff ist der intranasale tetravalente Impfstoff Fluenz Tetra ${ }^{\oplus}$

- Als Alternative intramuskulärer Impfstoff, in diesem Fall eventuell Verschiebung der Altersgrenze nach unten

- Rechtzeitige Information der impfenden Ärztinnen und in Ärzte

- Imagekampagne zur Erzielung einer adäquaten Beteiligungsrate

- Information der Öffentlichkeit frühzeitig, am besten schon jetzt in Verbindung mit Informationen zur Corona-Infektion und -Prävention

Erfreulicherweise wurde seitens des Ministeriums wenige Tage nach der Webkonferenz bereits veröffentlicht, dass für die Saison 2020/21 diese Maßnahmen wie besprochen umgesetzt werden sollen.

\section{Verfasser:}

Reinhold Kerbl
1 Kohlmaier B etal. (2020) BMC Public Health. https://doi.org/10.1186/ s12889-020-8239-2. 


\section{Österreichs Angebot an Zentren für Kinder- und Jugendrehabilitation}

Österreich hat sein Rehabilitationsnetz für Kinder und Jugendliche Ende 2019 um zwei weitere Zentren vergrößert: Rohrbach-Berg in Oberösterreich und Bad Erlach in Niederösterreich. Damit stehen mittlerweile 5 der 6 geplanten Rehablitationszentren für Kinder und Jugendliche mit bestimmten Erkrankungen in Österreich zur Verfügung (• Abb. 1).

„Viele Eltern kennen die Möglichkeit einer Reha-Maßnahme für ihr krankes Kind noch nicht. Eine mehrwöchige Rehamaßnahme kann aber den Gesundheitszustand von Kindern mit bestimmten Beschwerden deutlich verbessern, ihnen den Umgang mit einer chronischen Krankheit erleichtern, sie seelisch stärken und ihnen neue Perspektiven öffnen“, betont Prim.Univ.-Prof. Dr. Wolfgang Sperl, Vizepräsident der Österreichischen Gesellschaft für Kinder- und Jugendheilunde sowie Leiter des ÖGKJ-Referates Rehabilitation im Kindesund Jugendalter.

Ein Rehaaufenthalt ist für Kinder mit Erkrankungen des HerzKreislauf-Systems, der Lunge (z. B. Asthma), des Stoffwechsels (Angeborene Stoffwechselstörungen wie z. B. Phenylketonurie und Galaktosämie, Lysosomale Speicherkrankheiten, Diabetes mellitus), des Verdauungstraktes (Zöliakie, chronisch entzündliche Darmerkrankungen), mit psychischen oder psychiatrischen Erkrankungen und des Bewegungstraktes sowie bei einer Krebserkrankung erstattungsfähig.

\section{Unkomplizierte Antragstellung}

Befürwortet der Kinder- und Jugendarzt, ein anderer Arzt oder eine behandelnde Klinik eine Rehamaßnahme, muss dies zunächst in einem medizinischen Befundbericht begründet werden. Der Antrag zur Rehabilitation wird dann beim zuständigen Sozialversicherungsträger gestellt. Die entsprechenden Formulare können auf der Website der Österreichischen Sozialversicherung www.sozialversicherung.at oder www.hauptverband.at (https://www.sozialversicherung.at/cdscontent/load? contentid $=10008.650145 \&$ version $=1577724176$ ) ausgefüllt und heruntergeladen werden. Nach der Genehmigung erstatten die Krankenversicherungsträger die Kosten für die Rehabilitation in einem Kinder- und Jugendrehazentrum für den Patienten und eine Begleitperson.

\section{Keine Angst vor Unterrichtsausfall und Heimweh}

Die Angst, dass Kinder durch den mehrwöchigen Aufenthalt Unterricht versäumen, kann Eltern genommen werden. In der Regel erhalten Kinder in der Rehaeinrichtung Unterricht, der auf ihre Bedürfnisse zugeschnitten ist. Auch müssen Kinder nicht unter einer Trennung von ihren Eltern leiden, denn bis zu einem Alter von 12 Jahren können Eltern ihre Kinder begleiten. Sind die Kinder älter, besteht für Eltern meist die Möglichkeit, tageweise in der Umgebung untergebracht zu werden.

\section{Familienorientierten Rehabilitation - ein Meilenstein in der Kinder- und Jugendheilkunde}

In speziellen Fällen, wie bei einer Krebserkrankung, ist sogar eine sogenannte „familienorientierte Rehabilitation“(FOR) möglich, wie z.B. im Rehazentrum Leuwaldhof in St. Veit im Pongau. Eltern und die jungen Reha-Patienten sowie evtl. Geschwister können dort während des Rehaaufenthaltes in einem Familienappartment wohnen - und das ohne Zuzahlungspflicht für Minderjährige und Begleitpersonen. „Erste Forschungsergebnisse konnten belegen, dass Kinder sowohl körperlich als auch seelisch von einem Rehaufenthalt profitieren und sich ihre Lebensqualität deutlich verbessert. Und auch die Eltern kommen mit neuen Energien nach Hause zurück“, berichtet Prim.Univ.-Prof. Dr. Sperl, Leiter der Salzburger Universitätsklinik für Kinder- und Jugendheilkunde, die mit dem Leuwaldhof in St. Veit im Pongau wissenschaftlich zusammenarbeitet.

\section{Sechs Zentren}

Die sechs Rehazentren für Kinder und Jugendliche in Österreich haben sich auf bestimmte Fachgebiete spezialisiert, um Heranwachsende bestmöglich betreuen zu können (Abb 1).

- Abb. 1f: Das Rehazentrum Judendorf-Straßengel (Steiermark) mit 31 Betten liegt in der Nähe der Landeshauptstadt Graz und hat sich auf „mobilisierende Rehabilitation“" spezialisiert, insbesondere in den Bereichen Neuropädiatrie, Orthopädie, Neurochirurgie, Kinder- und Jugendchirurgie sowie Rheumatologie. Die Reha-Einrichtung versorgt vor allem junge Patienten aus dem Gebiet Österreich Süd (Kärnten, Steiermark, südl. Burgenland). Die Betreuung der Kinder beruht auf einem multimodalen Therapieprogramm, das z. B. neben der Förderung der Beweglichkeit (z. B. nach Operation) auch eine psychologische Betreuung sowie eine Ernährungsberatung beinhalten. Eltern können sich im Rahmen von Schulungen über die Krankheit ihres Kindes informieren. Kinder profitieren von modernen Verfahren, wie z.B. dem gewichtsentlastenden Gangtraining auf dem Unterwasserlaufband. Um während des drei- bis vierwöchigen oder längeren Aufenthalts keinen Lernstoff zu versäumen, gibt es einen für jedes Kind individuell zugeschnittenen Unterricht.

- Das Rehazentrum Wildbad-Einöd (Steiermark), zwischen Friesach in Kärnten und Neumarkt in der Steiermark gelegen, betreut Kinder mit Herz-Kreislauf- und Atemwegsproblemen (28 Plätze) sowie mit psychischen Problemen (24 Plätze) individuell und bedarfsgerecht. Um bei einem drei- bis fünfwöchigen Aufenthalt die Bildung nicht zu vernachlässigen, stehen auch hier ein Kindergarten und eine Heilstättenschule zur Verfügung.

- Der Leuwaldhof in St. Veit im Pongau (Salzburg) betreut Kinder mit Stoffwechselerkrankungen (angeborene Stoffwechselstörungen, Diabetes oder Adipositas), mit Erkrankungen des Verdauungssystems (wie Colitis ulcerosa, Morbus Crohn und Zöliakie, funktionelle Bauchschmerzen) und hämato-onkologischen (Blutkrebs) und immunologischen Erkrankungen. Bei letzten beiden Erkrankungen bietet die Rehaeinrichtung eine familienorientierte Reha (FOR) an (insgesamt 82 Betten). Experten kümmern sich im Rahmen eines ganzheitlichen und personalisierten Ansatzes bei ihren jungen Patienten sowohl um körperliche als auch um psychische Krankheitsfolgen. Wie in den anderen Rehaeinrichtungen gibt es eine integrierte Heilstättenschule (bis zur Oberstufe) und einen Kindergarten.

- Rohrbach-Berg (Oberösterreich) ist eines der nur für Kinder- und Jugendliche neu eröffneten Zentren (Eröffnung 10.09.2019). Es betreut schwerpunktmäßig Kinder mit HerzKreislaufkrankheiten, Krankheiten der Atemwege (z. B. Asthma), mit motorischen Problemen bzw. Störungen des 

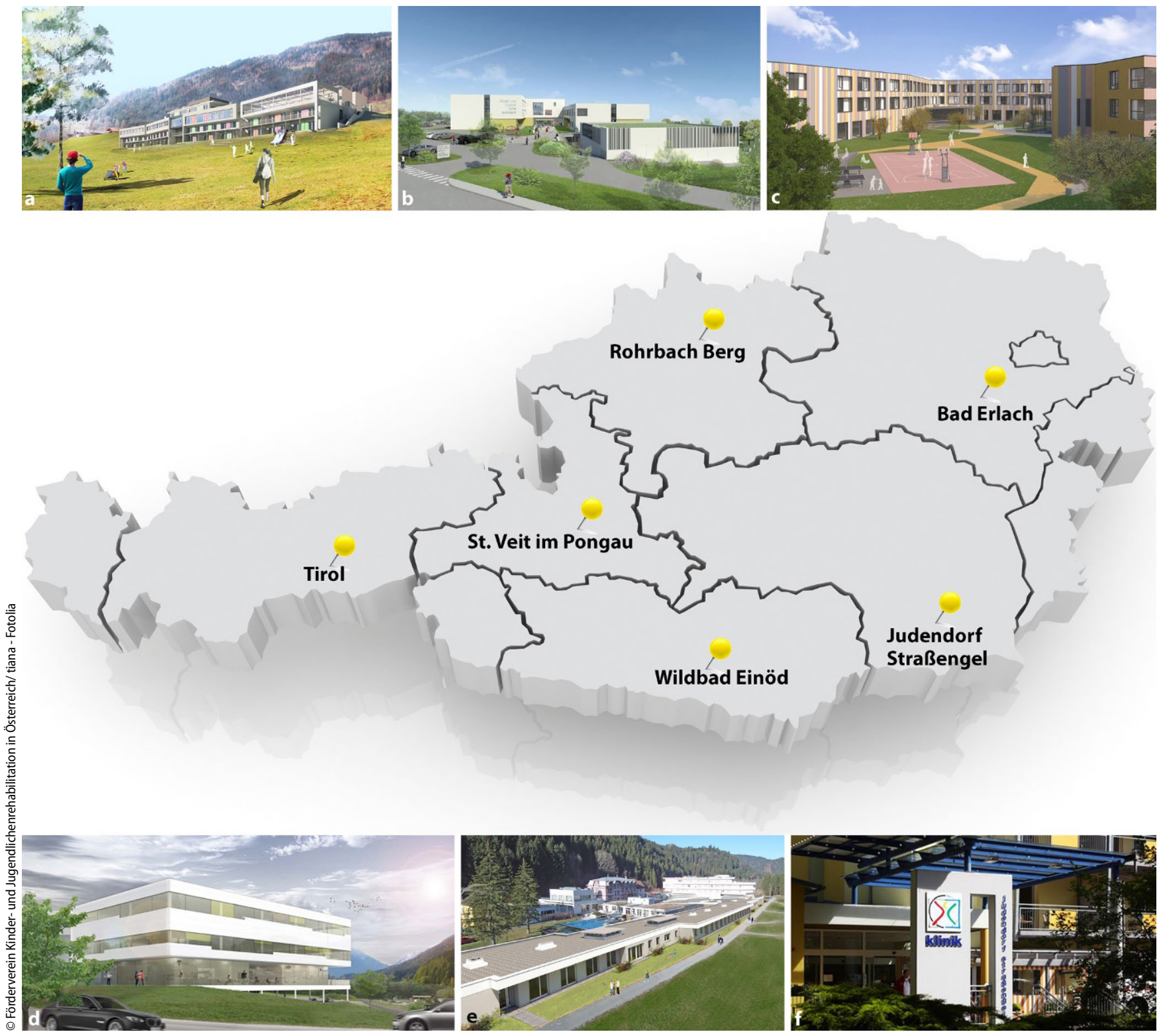

Abb. 1 ॥ Rehablitationszentren für Kinder und Jugendliche in Österreich a St. Veit im Pongau, Salzburg; b Rohrbach-Berg, Oberösterreich; c Bad Erlach, Niederösterreich; d Wiesing, Tirol; e Wildbad-Einöd, Steiermark; f Judendorf-Straßengel, Steiermark

Bewegungsapparates, neurologischen Krankheiten und psychiatrischen Erkrankungen (u. a. Essstörungen, Traumafolgestörungen, Autismus). 77 junge Patienten und 67 Begleitpersonen haben in der Einrichtung Platz. Ein multiprofessionelles Team betreut Kinder umfassend. Moderne Verfahren und altbewährte Therapien sorgen für eine optimale Behandlung, wie z. B. Wassertherapie und Stimulation der Muskeln mithilfe von Vibrationsplatten und -kipptischen. Eine Heilstättenschule gehört ebenso zur Einrichtung.

- Bad Erlach (Niederösterreich) behandelt nun seit dem 16.10.2019 Kinder mit Beeinträchtigungen des Bewegungsapparates, nach kinderchirurgischen oder neurochirurgischen Eingriffen. Auch bei neurologischen und psychiatrischen bzw. psychischen Problemen und Erkrankungen bietet diese Rehaeinrichtung Hilfe - mit 114 Betten. Kinder können dort ebenso einen Kindergarten besuchen und bekommen Schulunterricht. Die Nähe zur Wiener Neustadt erleichtert die Zusammenarbeit mit dem dortigen Landesklinikum und den Schulen vor Ort.

- Das Rehazentrum Wiesing (Tirol) ist für 2021 mit 22 Plätzen für eine mobilisierende Rehabilitation und 15 Betten für eine psychosoziale Rehabilitation geplant.

Weitere aktuelle Informationen rund um das Thema „Kindergesundheit" finden Sie auf der Internetseite der Österreichischen Gesellschaft für Kinder- und Jugendheilkunde (ÖGKJ) unter www.paediatrie.at und unter www.kinderaerzte-im-netz.at.

\section{Verfasserin: \\ Cornelia Steininger, Kinderärzte im Netz}




\section{Literatur}

1. Serie: Zentren für Kinder- und Jugendrehabilitation in Österreich (2019) Monatsschr Kinderheikd 167:86-88.

https://doi.org/10.1007/s00112-018-0643-7

2. Serie: Zentren für Kinder- und Jugendrehabilitation in Österreich (2019) Abteilung für Kinder- und Jugendrehabilitation/Klinik Judendorf-Straßengel - www. kinderreha.at. Monatsschr Kinderheikd 167:353-358. https://doi.org/10.1007/ s00112-019-0681-9

3. Jörgl P: Rehabilitation für Kinder und Jugendliche (2019) Die Klinik Judendorf Straßengel, Abteilung für Kinder- und Jugendrehabilitation im Indikationsbereich Mobilisierung stellt sich vor. Paediatr Paedolog 54:278-283. https://doi.org/10.1007/ s00608-019-00724-w

4. Serie: Zentren für Kinder- und Jugend-Rehabilitation (2019) In dieser Ausgabe stellt sich vor: Reha Wildbad-Einöd - Rehabilitation für Kardiologie/Pulmologie, Rehabilitation für Mental Health. Monatsschr Kinderheikd 167:658-659. https:// doi.org/10.1007/s00112-019-0724-2

5. Serie: Zentren für Kinder- und Jugend-Rehabilitation (2019) In dieser Ausgabe stellt sich vor: KinderJugendReha Leuwaldhof in Schwarzach/St. Veit im Pongau. Rahabilitation für Kinder und Jugendliche mit einer hämatol-onkologischen und immunologischen, einer Stoffwechselerkrankung oder einer Erkrankung des Verdauungssystems. Monatsschr Kinderheikd 167:949-654. https://doi.org/10.1007/ s00112-019-0779-w

6. Serie: Zentren für Kinder- und Jugend-Rehabilitation (2019) In dieser Ausgabe stellt sich vor: kokon, Einrichtung in Rohrbach-Berg für Rehabilitation für HerzKreislauferkrankungen und Pulmologie, Mobilisierung und Neurologie, Mental Health Monatsschr Kinderheikd 167:949-654. https://doi.org/10.1007/s00112019-0839-1

7. Kokon. Rehabilitation für Kinder und Jugendliche. https://kokon.rehab/. Zugegriffen: 23. Jan 2020

8. Der Gesundheit verpflichtet. Familienorientierte Kinder-Rehabilitation einzigartig in Österreich. Das österreichische Gesundheitswesen - ÖKZ 2019; 03-04 (60), 42-43. http://www.schaffler-verlag.com/pdf-download?pdf=32413

\section{Geburtstage}

Die Österreichische Gesellschaft für Kinder- und Jugendheilkunde gratuliert herzlich ihren Mitgliedern, die im nächsten Monat einen „besonderen" Geburtstag feiern:

\section{Jahre und älter werden:}

17.07.1930 | Dr. Gottfried Harasek, Wien

24.07.1936 | MR Dr. Laszlo Kiraly, Wien

\section{Jahre wird:}

26.07.1945 | Dr. Ingmar Gaßner, Innsbruck

\section{Jahre wird:}

24.07.1950 | MR Dr. Waltraud Schobermayr, Vöcklabruck

\section{Jahre werden:}

04.07.1955 | Dr. Kurt Gremel, Wien

11.07.1955 | Univ. Prof. Dr. Oskar Haas, Wien

17.07.1955 | Dr. Karl Gutenberger, Zams

\section{4. Österreichische Jahrestagung für Pädiatrische Psychosomatik}

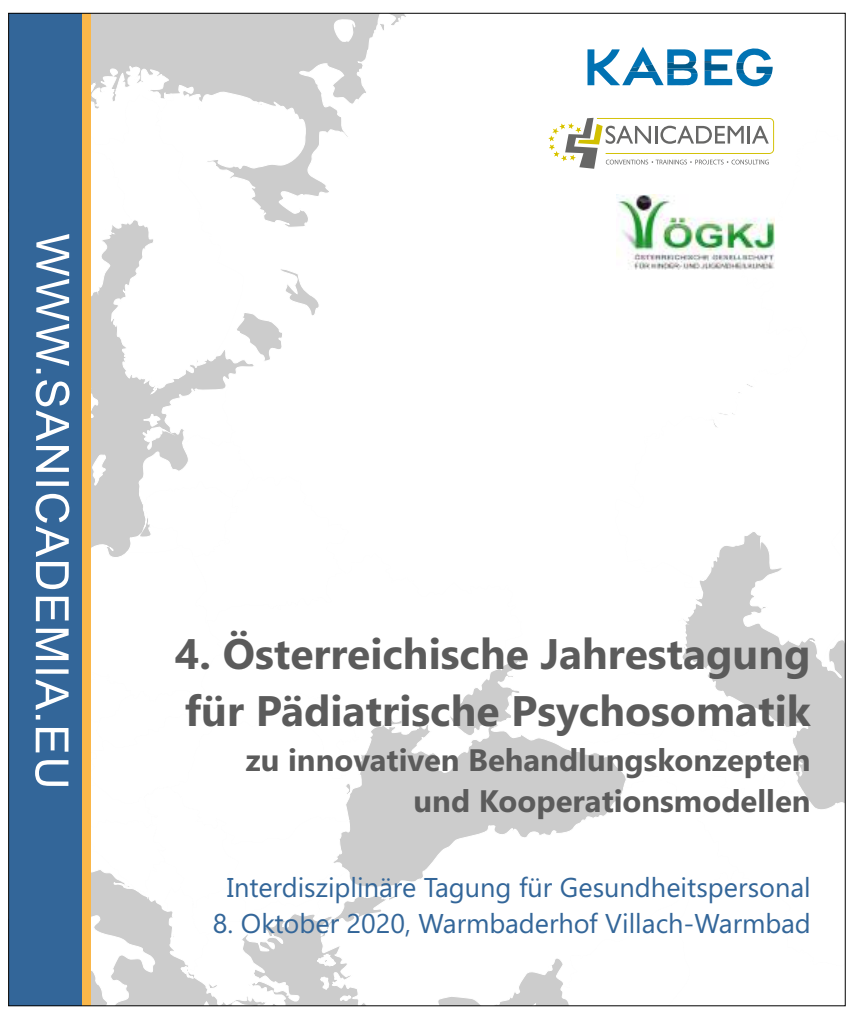

\begin{tabular}{|c|c|}
\hline & PROGRAMM FREITAG 08.10.2020 \\
\hline 08:00 - 09:00 & REGISTRIERUNG \& GET TOGETHER \\
\hline \multirow[t]{2}{*}{ 09:00 - 09:05 } & $\begin{array}{l}\text { Begrüßung und feierliche Eröffnung } \\
\text { Robert Birnbacher (Landeskrankenhaus Villach), } \\
\text { Daniela Karall (OGKJ), } \\
\text { Beate Prettner (Kärntner Landesregierung), } \\
\text { Arnold Gabriel (KABEG) }\end{array}$ \\
\hline & Vorsitz: Adrian Kamper, Grieskirchen/Reinhold Kerbl, Leoben \\
\hline 09:45 - 10:15 & $\begin{array}{l}\text { Psychische Gesundheit bei Österreichs Kindern und Jugendlichen } \\
\text { Rosemarie Felder-Puig, Wien }\end{array}$ \\
\hline $10: 15-10: 45$ & $\begin{array}{l}\text { Psychodiabetologie als neues Fachgebiet } \\
\text { G. Berger, Wien }\end{array}$ \\
\hline $10: 45-11: 15$ & $\begin{array}{l}\text { Psychodiabetologie am LKH Villach } \\
\text { Eva Schlintl, Villach }\end{array}$ \\
\hline \multirow[t]{2}{*}{$11: 15-11: 45$} & KAFFEEPAUSE \\
\hline & Vorsitz: Daniela Karall, Innsbruck/Claudia Klier, Wien \\
\hline $11: 45-12: 15$ & $\begin{array}{l}\text { Konzepte in der Behandlung von Bindungsstörungen } \\
\text { Augustine Gasser, Villach }\end{array}$ \\
\hline $12: 15-12: 45$ & $\begin{array}{l}\text { Adäquate Unterstützung von Kindern psychisch erkrankter Eltern } \\
\text { Ingrid Zechmeister-Koss }\end{array}$ \\
\hline $12: 45-13: 15$ & $\begin{array}{l}\text { Kinderschutz } \\
\text { Reinhold Kerbl, Leoben }\end{array}$ \\
\hline \multirow[t]{2}{*}{$13: 15-14: 45$} & MITTAGSPAUSE \\
\hline & Vorsitz: Robert Birnbacher, Villach/N.N. \\
\hline $14: 45-15: 15$ & $\begin{array}{l}\text { Kunsttherapie und deren Einsatz in der Kinder- und Jugend- } \\
\text { psychosomatik im Klinikum Wels-Grieskirchen } \\
\text { Adrian Kamper, Wels } \\
\text { Karin Tikal, Wels }\end{array}$ \\
\hline 15:15 - 15:45 & $\begin{array}{l}\text { Ist Magersucht bei ausreichender Ernährung und damit Erreichen eines } \\
\text { adäquaten Gewichts geheilt? } \\
\text { Victor Aguiriano Moser, Graz }\end{array}$ \\
\hline 15:45 - 16:30 & REFLEXION und DISKUSSION \\
\hline
\end{tabular}




\section{HINWEIS:}

Das Nationale Zentrum Frühe Hilfen (NZFH.at) bietet diese online verfügbaren Fortbildungen zu Frühen Hilfen an:

siehe www. fruehehilfen.at/fortbildung.htm

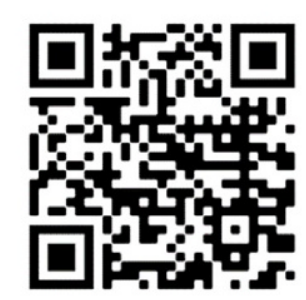

\section{FrïheHILFEN}

\section{Gesundheit Österreich \\ $\mathrm{GmbH} \bullet \bullet$}
= Bundesministerium Soziales, Gesundheit, Pflege und Konsumentenschutz

VORSORGEMITTEL DER BUNDESGESUNDHEITSAGENTUR

IMPRESSUM: Gesundheit Österreich $\mathrm{GmbH}$, Nationales Zentrum Frühe Hilfen. Layout: NZFH.at Coverfoto: @ NZFH.at, Bruckmüller. Wien 2020

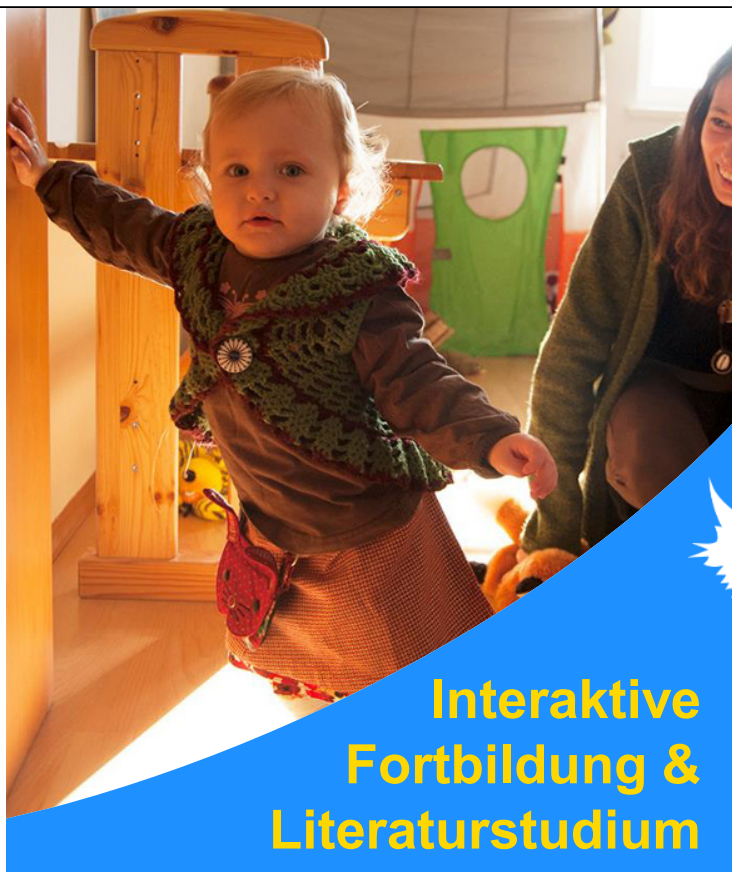

Frühe Hilfen verbessern die Entwicklungsmöglichkeiten und Gesundheitschancen von Kindern und deren Eltern frühzeitig und nachhaltig. Als frühe präventive Maßnahme wirken sie positiv auf den körperlichen und seelischen Gesundheitszustand von Kindern und Jugendlichen und legen den Grundstein für Gesundheit im Erwachsenenalter.

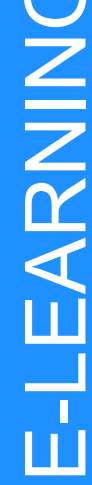

Um Familien mit Unterstützungsbedarf zu erreichen, braucht es einen aktiven und systematischen Zugang über Fachkräfte und Institutionen, die mit schwangeren Frauen und Müttern bzw. Familien mit Kleinkindern in Kontakt sind. Im Zeitraum von Schwangerschaft, Geburt und früher Kindheit kommt dem Gesundheitsbereich eine besondere Rolle zu, da nahezu alle Familien Gesundheitsdienstleistungen in Anspruch nehmen. Die regionalen Frühe-HilfenNetzwerke streben daher eine enge Kooperation mit dem medizinischen Bereich an und richten sich insbesondere an Krankenhäuser (v. a. Geburtenstationen) sowie niedergelassene Gesundheitsberufe mit Fokus auf Gynäkologie, Pädiatrie, Allgemeinmedizin und Hebammen. 


\section{Negative \\ Kindheitserfahrungen, toxischer Stress und Frühe Hilfen}

\section{LITERATUR- STUDIUM}

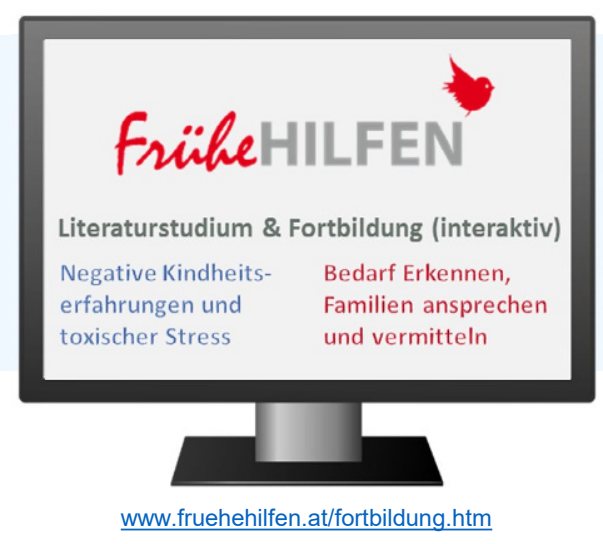

\section{Frühe Hilfen - Bedarf erkennen,} Familien ansprechen und vermitteln

\section{INTERAKTIVE FORTBILDUNG}

\section{Das Literaturstudium bietet ...}

- Aktuelle Evidenz zu den gesundheitlichen Folgen von negative Kindheitserfahrungen

- Wissen zu den (neurobiologischen) Auswirkungen der Erfahrung von toxischem Stress auf die Entwicklung des Gehirns und die langfristige Gesundheit

- Darstellung der zentrale Aspekte des österreichischen Angebots der Frühen Hilfen als transgenerationales Programm zur Unterstützung in der frühen Kindheit

- Informationen und Materialien, die das Erkennen und Ansprechen von relevanten Belastungen und die Vermittlung an Frühe Hilfen unterstützen

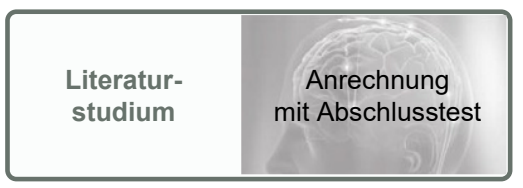

6 DFP-Punkte für Ärztinnen/Ärzte 15 Fortbildungspunkte für Hebammen
Die interaktive Fortbildung bietet ...

- Präsentation der zentralen Elemente des Literaturstudiums auf 45 Folien zum Durchklicken

- Praxisbeispiele, integrierte Videos mit einer Zusammenfassung der wesentlichen Inhalte und Quizze zur Selbstüberprüfung des gelernten Wissens

- Links zu den unterstützenden Materialien und weiterführenden Infos

Arbeitsunterlagen, Gesprächsleitfäden \& Co siehe www.fruehehilfen.at/arbeitsunterlagen.htm
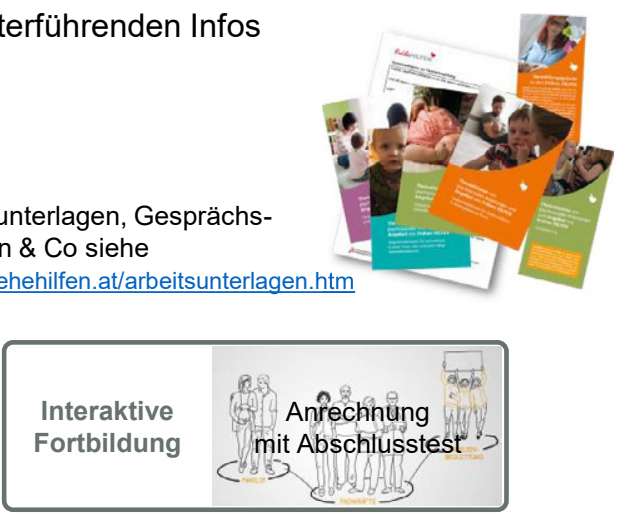

1 DFP-Punkt für Ärztinnen/Ärzte 3 Fortbildungspunkte für Hebammen

\section{ÖGKJ-Geschäftsstelle Öffentlichkeitsarbeit und} Standesführung

\section{c/o S12! studio12 gmbh, Ralph Kerschbaumer}

Kaiser Josef Straße 9, A-6020 Innsbruck

Tel.: +43 (0) 512-89 04 38, Fax: +43 (0) 512-89 04 38-15

E-Mail: office@paediatrie.at, www.paediatrie.at

Alle Informationen zur Anmeldung bei der ÖGKJ finden Sie unter

www.paediatrie.at/mitgliedschaft. 